\title{
A PCR-Derived Library of Random Point Mutations within the V3 Region of Simian Immunodeficiency Virus
}

\author{
Frank Kirchhoff and Ronald C. Desrosiers
}

New England Regional Primate Research Center, Harvard Medical School, Southborough, Massachusetts $01772-9102$

\begin{abstract}
Oligonucleotide primers corresponding to variable region 3 (V3) of simian immunodeficiency virus (SIV) were randomly mutagenized during synthesis by doping each of the four nucleoside phosphoramidites with a small amount of the other three. PCR was then used to incorporate the altered sequences into larger, clonable DNA fragments by spliced overlap extension (SOE). With the composition of the phosphoramidites used, 53 of the 100 clones analyzed were unique, having one or more point mutation within the 84-bp target sequence. These $\mathbf{5 3}$ unique clones contained an average of 2.1 nucleotide substitutions and 1.5 amino acid substitutions per clone within the target V3 sequence. Of the internal 25 amino acid positions within the V3 domain, 23 were changed at least once. This method should be generally useful for the construction of libraries of random point mutations within a defined target DNA sequence.
\end{abstract}

A g the methods that have been for the specific mutagenesis of DNA sequences, PCR is one of the most powerful. ${ }^{(1-6)}$ It allows rapid preparation of relatively large quantities of mutated DNA with the use of mutated oligonucleotide primers. Because of the error frequency of Taq and other polymerases used in PCR, it is generally most convenient to use PCR-based mutagenesis strategies which minimize the length of the PCR-derived DNA, ${ }^{(1-3)}$ rather than amplifying entire plasmids. ${ }^{(4-7)}$ Recently, Morrison and Desrosiers used PCR with spliced overlap extension (SOE) in the presence of mixed populations of oligonucleotide primers to mutagenize selected sites in a target DNA sequence. ${ }^{(7)}$

In some cases, it is desirable to analyze an extensive collection of mutations over the full length of a defined target DNA sequence. Hutchinson et al. described the generation of a library containing all possible point mutations within a 30-bp target sequence. ${ }^{(8)}$ They doped each of the four nucleoside phosphoramidites with a small amount of the other three and cloned the two complementary mutagenic oligonucleotides bearing cohesive ends directly into an M13 vector. This specific approach does not have general utility because of the need for conveniently located restriction sites at the ends of the target sequence. However, it did set the stage for development of protocols for extensive mutagenesis of target DNA sequences in phage vectors. ${ }^{(9,10)}$

Here, we present a method that combines the advantages of the SOE PCR method with the doping procedure originally used by Hutchinson et al. for the generation of potentially complete libraries of point substitutions. This procedure is likely to be generally useful for saturation mutagenesis of small target regions. Because it is easy to generate a large number of variants in a defined area with this method, it may also be useful for biological selection experiments.

\section{MATERIALS AND METHODS Oligonucleotide Synthesis}

Oligonucleotides were synthesized with a Cyclone DNA synthesizer (Milligen-Biosearch, Burlington, MA). Prior to synthesis, each vial of phosphoramidite was doped with $1.5 \%(\mathrm{vol} / \mathrm{vol})$ of each of the other three. The primers were purified on oligonucleotide purification cartridges (Applied Biosystems Inc., Foster City, CA). The four mutagenic primers that were used span nucleotides 7535 7618 of the simian immunodeficiency virus (SIV)mac239 sequence. $^{(11)}$ The primer sequences are shown in Table 1. The expected number of nucleotide changes per oligonucleotide was calculated on the basis of the concentration of the other three nucleotides $[4.5 \%$ (vol/ vol)] versus the concentration of the wild-type nucleotide [ $95.5 \%$ ( $\mathrm{vol} / \mathrm{vol})]$.

\section{PCR Conditions}

Twenty-five nanograms of the p316EM * plasmid served as the template for PCR amplification; p316EM * contains the env gene of a macrophage tropic variant of the SIVmac239 clone. ${ }^{(12)}$ Reactions were performed using a DNA thermal cycler (Perkin-Elmer Cetus, Norwalk, CT) and the manufacturer's PCR reagent kit. 
TABLE 1 Oligonucleotides Used for Mutagenesis of the SIVmac V3 Region

\begin{tabular}{lllc}
\hline Primer & Sequence $\left(5^{\prime} \rightarrow 3^{\prime}\right)$ & $\begin{array}{l}\text { Position in } \\
\text { SIVmac239 } \\
\text { sequence }\end{array}$ & $\begin{array}{l}\text { Expected number of } \\
\text { nucleotide changes } \\
\text { per oligonucleotide }\end{array}$ \\
\hline F5 & CCACTGTAACACTTCTG & $7230 \rightarrow 7246$ & 0 \\
F6 & CTGTATTCCTATCTTCTACC & $7852 \rightarrow 7833$ & 0 \\
F7 & GGTGACTGGTAAAACTGTCTTATTTCCTGGTCTTCTAC & $7572 \rightarrow 7535$ & 1.7 \\
F8 & AGACAGTTTTACCAGTCACCATTATGTCTGGATTGGTTTCC & $7553 \rightarrow 7594$ & 1.9 \\
F9 & GGAAAACCAATCCAGACATAATGGTGACTGGTAAAACTGTC & $7594 \rightarrow 7554$ & 1.8 \\
F10 & TATGTCTGGATTGGTTTCCACTCACAACCAATCAATGATAGGC & $7575 \rightarrow 7618$ & 2.0 \\
\hline
\end{tabular}

For the synthesis of oligonucleotides F7-F10, 1.5\% (vol/vol) of each of the other phosphoramidite nucleoside mixes was added to each vial. Thus, the probability for a change at each position is $4.5 \%$.

The PCR mutagenesis strategy is shown schematically in Figure 1. The first round of amplification using primer pairs $F 5 / 7$, F5/9, F6/8, or F6/10 was per-

formed in a $100-\mu$ l volume using 60 pmoles of each primer (25 cycles, 1 min at $94^{\circ} \mathrm{C}, 1 \mathrm{~min}$ at $45^{\circ} \mathrm{C}, 1 \mathrm{~min}$ at $68^{\circ} \mathrm{C}, 2.5$ $\mathrm{mM} \mathrm{MgCl}_{2}$ ). Thereafter, $25 \mu \mathrm{l}$ of the PCR

\section{"V3" region}

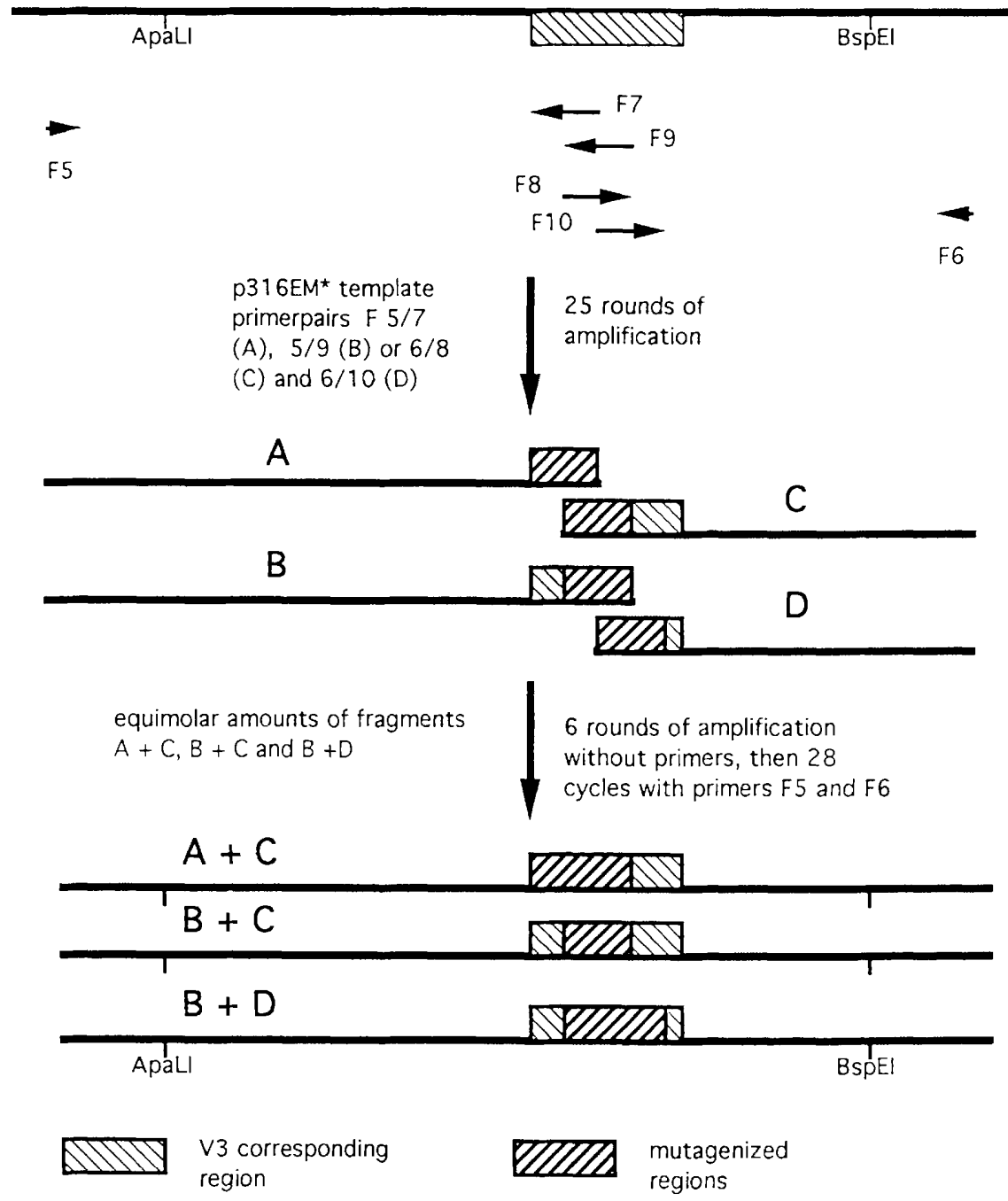

FIGURE 1 Strategy for random mutagenesis of the SIVmac V3 region. The p316EM* plasmid was used as a template for amplification with doped oligonucleotides (see Table 1). First round, PCR-generated DNA fragments A-D were subsequently used for the synthesis of DNA fragments containing random changes in the $\mathrm{V} 3$ region at three slightly different locations, as indicated. products was analyzed by electrophoresis in a $1.5 \%$ agarose gel. PCR products of the expected size, ranging from 280-362 $\mathrm{bp}$, were isolated with the GeneCleanII kit (BIO101 lnc., La Jolla, CA). To generate a linear double-stranded template, 0.1 pmole of the left and right half fragments were combined and six cycles of amplification without any additional primer were performed. Then, 50 pmoles of outer primers pF5 and pF6 were added and another 28 cycles of amplification were performed to generate the three classes of 622-bp products, which differ slightly in the positions of mutations (Fig. 1).

\section{Cloning and DNA Sequencing}

The PCR products were isolated from agarose gels and digested with ApaLI and $B s p E I$. Because three ApaLI sites are present in the p316EM* plasmid, a gel purified subfragment of $\mathrm{p} 316 \mathrm{EM}^{*}$ was used for insertion of the mutant PCR products. The $\mathrm{p} 316 \mathrm{EM}$ * vector DNA was obtained after transformation of the $\mathrm{dam}^{-}$strain of Escherichia coli JM110 (Stratagene, La Jolla, CA). The unmethylated $\mathrm{p} 316 \mathrm{EM}$ * DNA was digested with $B s p \mathrm{EI}$, dephosphorylated, and digested with SphI. The 7.7-kbp restriction fragment was isolated and ligated to the dephosphorylated $0.85-\mathrm{kpb} S p h \mathrm{I} / A$ paLI restriction fragment derived from the same vector. The 8.6-kbp ligation product was isolated from an agarose gel, ligated to a fivefold molar excess of an equimolar mixture of the three different 457-bp ApaLI/BspEI PCR fragments, and transformed into supercompetent $E$. coli XL-1 Blue cells (Stratagene). About 10\% of the transformation mix was spread on LB-Amp plates in serial dilutions, and the rest was used directly for plasmid 
DNA preparation. The resulting plasmid products varied considerably in size and 95\% were smaller than expected. Plasmids migrating at the correct size were purified from an agarose gel and retransformed into $E$. coli XL-1 Blue. Plasmid DNA was prepared from single colonies, and the V3 target sequence was determined. For a subset of clones containing changes within $V 3$, the entire sequence between the ApaLI and BspEI sites was determined.

Serial dilutions of the transformation mixture showed that the mixed plasmid population represented $\sim 4000$ colonies. Agarose gel analysis revealed that only $\sim 5 \%$ of the plasmid population obtained had the correct size. Therefore, the plasmid population used to retransform $E$. coli after the purification step represented only $\sim 200$ different PCR fragments. The low proportion of correctly sized plasmids obtained after the first round of transformation was probably related to the unmethylated status of the vector DNA. In recent experiments using normally methylated p316EM* DNA cut with MroI (Boehringer-Mannheim) instead of BspEI, $\sim 90 \%$ of the plasmid population after initial transformation had the correct electrophoretic mobility on an agarose gel. In addition, cloning efficiencies were $\sim 20$-fold higher than in the initial experiment.

\section{RESULTS AND DISCUSSION}

The analysis of 105 single colonies showed that 100 of them contained the insertion of a $0.46-\mathrm{kbp}$ restriction fragment. Sequencing of the variable region 3 (V3) target region within these 100 clones showed that 69 contained at least
TABLE 2 Numbers of Amino Acid and Nucleotide Substitutions in the 53 Unique V3 Mutant Clones

\begin{tabular}{lc}
\hline $\begin{array}{l}\text { Number of nucleotide } \\
\text { changes/number of } \\
\text { amino acid changes }\end{array}$ & Number of clones \\
\hline $1 / 0$ & 10 \\
$1 / 1$ & 10 \\
$2 / 1$ & 9 \\
$2 / 2$ & 9 \\
$3 / 1$ & 1 \\
$3 / 2$ & 4 \\
$3 / 3$ & 1 \\
$4 / 2$ & 2 \\
$4 / 3$ & 4 \\
$4 / 4$ & 1 \\
$5 / 4$ & 2 \\
Total & 53 \\
\hline
\end{tabular}

one nucleotide change from the parental. Of these 69 clones, 53 had a unique sequence (Table 2). Eight sequences were present twice and four sequences were present three times in the plasmid population.

Among the 53 unique clones, there were 112 nucleotide substitutions and 77 predicted amino acid substitutions (Table 2); thus, the average number of nucleotide substitutions per V3 target sequence was 2.1 per clone, and the average number of translated amino acid substitutions per V3 target sequence was 1.5 per clone. If one assumes no selection against incorporation of nucleotide substitutions into cloned DNA, we would have expected an average of 1.9 nucleotide substitutions per clone. This expectation is based on an average of 1.9 nucleotide misincorporations per singlestranded oligonucleotide primer (Table
1), 3.8 single-stranded nucleotide substitutions per double-stranded DNA fragment, and it assumes equidirectional resolution to 1.9 matched base-pair substitutions per clone by the PCR process and mismatch repair in $E$. coli. Thus, the 53 unique clones had an average nucleotide substitution frequency (2.1) that was close to what would be expected (1.9) based on these assumptions. If, however, one includes the total 100 clones, 31 of which had no substitutions at all in the target sequence, the observed frequency was somewhat less than expected. Other investigators have observed a somewhat lower than expected mutation frequency among the final clones analyzed following oligonucleotide doping. ${ }^{(7,8)}$ Inefficient PCR amplification with mismatched primers and inefficient survival of mismatched sequences in $E$. coli may contribute to a lower than expected frequency of nucleotide substitutions in the final clones. PCR amplification may be especially inefficient when the sequences near the $3^{\prime}$ end of the primer are not perfectly matched.

Despite the fact that the 84-bp target sequence was AT rich (59.5\%), 73 of the 112 nucleotide substitutions $(65 \%)$ were changes to A or T (Fig. 2). Hutchinson et al. ${ }^{(8)}$ also observed a higher than expected frequency of changes to $A$ or $T$. The reasons for this are not clear but could possibly involve the kinetics of phosphoramidite condensation during oligonucleotide synthesis.

Of the 53 mutant clones, 20 had a single nucleotide change, 18 had two changes, 6 had three changes, 7 had four changes, and 2 had five changes in the V3 target sequence (Table 2 ). Two clones

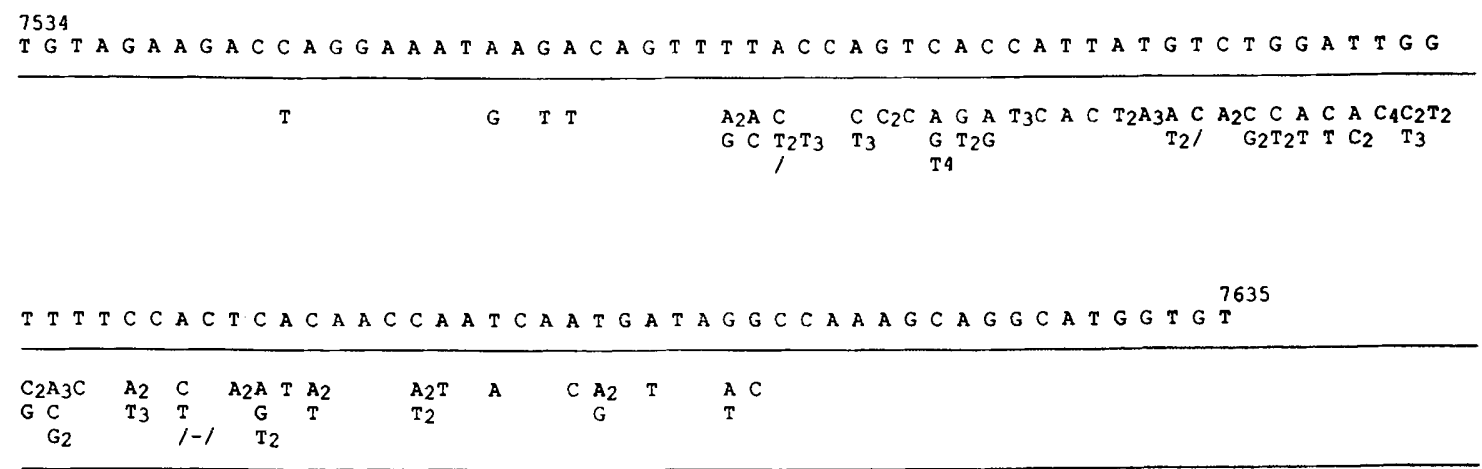

FIGURE 2 Nucleotide changes in the SIVmac p316EM*V3 region resulting from PCR-directed mutagenesis. Numbers indicate the frequency of mutations if greater than one. (/) A single nucleotide deletion; $(/-)$-a double nucleotide deletion. The complete length of the V3 cysteine loop is shown. The target sequences for mutagenesis include bases 7535-7618. 


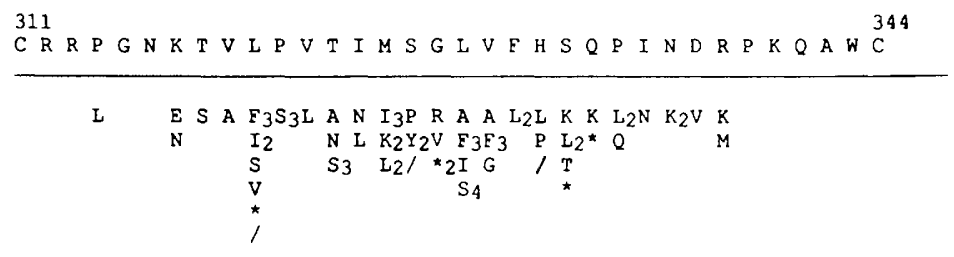

FICURE 3 Amino acid changes in the SIVmac p316EM* V3 region resulting from PCR-directed mutagenesis. Numbers indicate frequency of the mutation when greater than one. ( $\left.{ }^{*}\right)$ Change to a stop condon. (/) Location of nucleotide deletions resulting in a frameshift of downstream sequences.

had a deletion of a single nucleotide and one clone had a deletion of two nucleotides (Fig. 2). The use of PCR fragments $A$ and $\mathrm{C}, \mathrm{B}$ and $\mathrm{D}$, and $\mathrm{A}$ and $\mathrm{D}$ allowed nucleotide substitution over the full 84 bp of target sequence (Fig. 1). This procedure was also intended to obtain higher frequencies of point mutations within the central part of the V3 region due to the overlapping nature of the mutagenic F7, F8, F9, and F10 primers (Fig. 1). A higher frequency of mutations was concentrated in this central region (Figs. 2 and 3). This basic mutagenesis strategy can be easily modified to distribute point mutations in any manner desired.

The vast majority of changes occurred in the region covered by the mutagenic oligonucleotides. However, eight additional changes, which were most likely due to errors by the Taq polymerase, were identified elsewhere in the $0.46-\mathrm{kb}$ PCR fragments analyzed. This random substitution rate was $\sim 1$ per 3300 bp and, thus, similar to the rate of 1 per 4000 bp reported by Ho et al. ${ }^{(1)}$ and much lower than the 1 per 840 bp found by Morrison and Desrosiers. ${ }^{(7)}$

For the random mutagenesis of short target regions within longer DNA sequences, the method described here has some advantages over those described previously. ${ }^{(1,2,7,8)}$ The doped oligonucleotide synthesis, PCR, and cloning steps are easy to perform and require no unusual advanced preparation. The average number of mismatches in each oligonucleotide and any preference for desired changes (e.g., by adding only one additional nucleoside solution) can easily be varied. The choice of the external primers makes it possible to minimize the size of the mutated PCR fragment and also makes it easy to reclone the mutated fragments into longer sequences.

The retrieval of 53 unique mutants from a population of 100 clones shows that this approach is useful for the ex- tensive random mutagenesis of defined target sequences. PCR fragments obtained with this mutagenesis strategy potentially contain all possible point substitution mutations. With high cloning efficiencies, this approach can also be useful for biological selection experiments. For example, we have used mixed plasmid populations containing a high number of changes within V3 to select identify changes within V3 that can compensate for defects in other regions of the SIV envelope.

\section{ACKNOWLEDGMENTS}

We thank Hilary G. Morrison and Dean Regier for their helpful advice. This work was supported by U.S. Public Health Service grants AI 25328 and RR00168, and by a fellowship of the Deutsches Krebsforschungszentrum to F.K.

\section{REFERENCES}

1. Ho, S.N., H.D. Hunt, R.M. Horton, J.K. Pullen and L.R. Pease. 1989. Site-directed mutagenesis by overlap extension using the polymerase chain reaction. Gene 77: 51-59.

2. Horton, R.M., H.D. Hunt, S.N. Ho, J.K. Pullen, and L.R. Pease. 1989. Engineering hybrid genes without the use of restriction enzymes: Gene splicing by overlap extension. Gene 77: 61-68.

3. Higuchi, R., B. Krummel, and R.K. Saiki 1988. A general method of in vitro preparation and specific mutagenesis of DNA fragments: Study of protein and DNA interactions. Nucleic Acids Res. 16: 73517367.

4. Jones, D.H., K. Sakamoto, R.L. Vorce, and B.H. Howard. 1990. DNA mutagenesis and recombination. Nature 344: 793794. Cortopassi, and D.J. Galas. 1989. A simple method for site-directed mutagenesis using the polymerase chain reaction. $\mathrm{Nu}$ cleic Acids Res. 17: 6545-6551. variants with altered cell tropism and to

5. Hemsley, A., N. Arnheim, M.D. Toney, G.
6. Jones, D.H. and B.H. Howard. 1991. A rapid method for recombination and sitespecific mutagenesis by placing homologous ends on DNA using polymerase chain reaction. BioTechniques 10: 62-66.

7. Morrison, H.G. and R.C. Desrosiers. 1993. A PCR-based strategy for extensive mutagenesis of a target DNA sequence. BioTechniques 14: 454-457.

8. Hutchinson, C.A., S.K. Nordeen, K. Vogt, and M.H. Edgell. 1986. A complete library of point substitution mutations in the glucocorticoid response element of mouse mammary tumor virus. Proc. Natl. Acad. Sci. 83: 710-714.

9. Zoller, M.J. and M. Smith. 1987. Oligonucleotide-directed mutagenesis: A simple method using two oligonucleotide primers and a single-stranded DNA template. Methods Enzymol. 154: 329-350.

10. Loeb, D.D., R. Swanstrom, L. Everitt, M. Manchester, S.E. Stamper, and C.A. Hutchinson IlI. 1989. Complete mutagenesis of the HIV-1 protease. Nature 340: $397-400$.

11. Regier, D.A. and R.C. Desrosiers. 1990. The complete nucleotide sequence of a pathogenic molecular clone of simian immunodeficiency virus. AIDS Res. Hum. Retroviruses 6: 1221-1231.

12. Mori, K., D.J. Ringler, T. Kodama, and R.C. Desrosiers. 1992. Complex determinants of macrophage tropism in env of SIV. J. Virol. 66: 2067-2075.

Received December 14, 1992; accepted in revised form on February 22, 1993. 


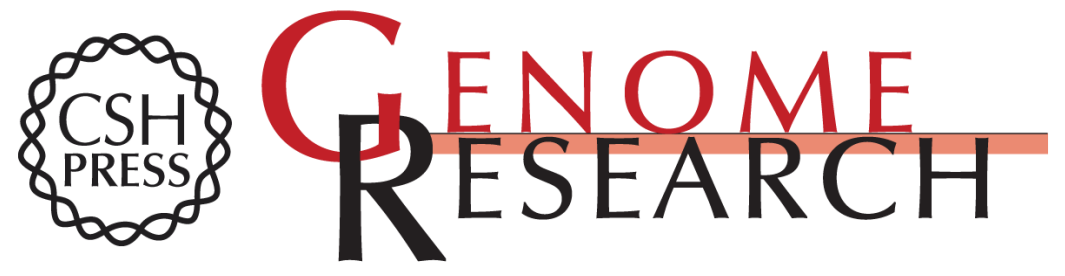

\section{A PCR-derived library of random point mutations within the V3 region of simian immunodeficiency virus.}

F Kirchhoff and R C Desrosiers

Genome Res. 1993 2: 301-304

Access the most recent version at doi:10.1101/gr.2.4.301

References This article cites 12 articles, 2 of which can be accessed free at:

http://genome.cshlp.org/content/2/4/301.full.html\#ref-list-1

\section{License}

Email Alerting Receive free email alerts when new articles cite this article - sign up in the box at the Service top right corner of the article or click here.

\section{Affordable, Accurate Sequencing.}

To subscribe to Genome Research go to:

https://genome.cshlp.org/subscriptions 